\title{
Amniotic Band Sequence - A Debilitating Syndrome
}

\author{
Uma R, ${ }^{1}$ Garg A, Patnaik SK' \\ 'Department of Pediatrics, 7 Air Force Hospital, Kanpur Cantt, 91 208004, India.
}

A male baby was delivered by Caesarean section at term to a 35 years second gravida lady with fibroid uterus. The mother was booked and immunized and not on any medication. Antenatal and natal periods including serial ultrasounds were normal. At birth the neonate was observed to have multiple grooves over ring \& middle index finger of left hand and hypoplasia of left little finger. Both great toes were amputated. In view of asymmetrical involvement, presence of amniotic bands, no other organ involvement and sporadic nature, he was diagnosed as a case of amniotic band sequence. Congenital amputations should be distinguished from congenital hypoplasia and/or aplasia. Differentiation is important for genetic counselling and evaluation of the risk of recurrence.

Keywords: Amniotic Bands, Intrauterine Amputation, Intrauterine Constriction Rings.

\section{INTRODUCTION}

Amniotic Band Syndrome (ABS) is a sporadic constellation of congenital malformations attributed to amniotic bands. ${ }^{1-3}$ These entangle fetal parts during intrauterine life, which results in a broad spectrum of anatomic disturbances - ranging from minor constriction rings to complex, bizarre multiple congenital anomalies incompatible with life. ${ }^{3}$ Most commonly the extremities are affected. Its incidence ranges from 1 in 10,000 45,000 births with pregnancy losses occurring in $1.8 \%$ cases. ${ }^{4-6}$ We report a case with severe manifestations of $A B S$.

\section{CASE REPORT}

A male baby was delivered operatively at term to a 35 years second gravida with fibroid uterus. The mother was booked and immunized and not on any medication. VDRL was negative. Antenatal and natal periods were uneventful. Serial antenatal ultrasound examinations did not reveal any abnormality. At birth there was no asphyxia and weight was $3.5 \mathrm{kgs}$. The umbilical cord was normal with three vessels. Systemic examination was essentially normal. General physical examination revealed multiple grooves over ring \& middle index 
finger of left hand and hypoplasia of left little finger. Distal and middle phalanx of left middle finger was gangrenous with amniotic bands at the base (Figure 1). Both great toes were amputated (Figure 2). Routine screening for other anomalies were normal. Skeletal survey and ultrasound examination of the abdomen and CT scan of head did not reveal any abnormality. Pedigree analysis was non-contributory.

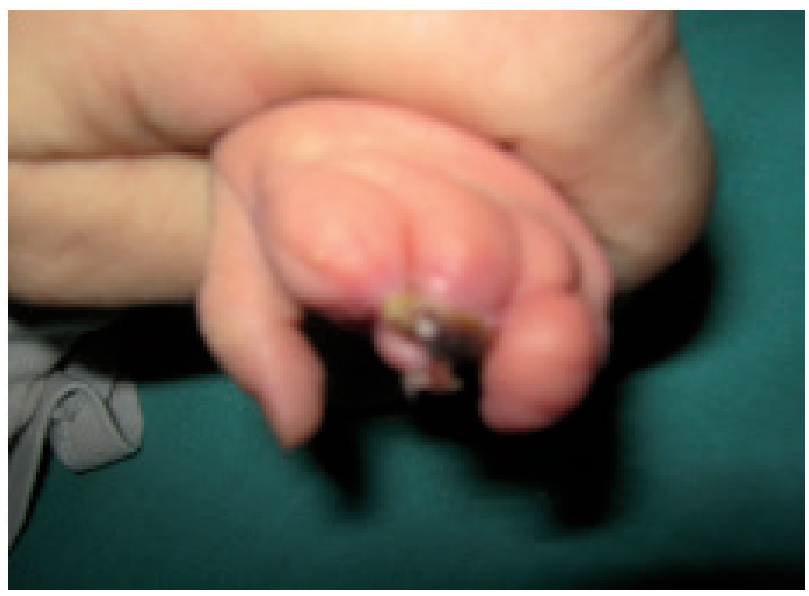

Figure 1. Distal and middle phalanx of left middle finger was gangrenous with amniotic constriction bands at the base.

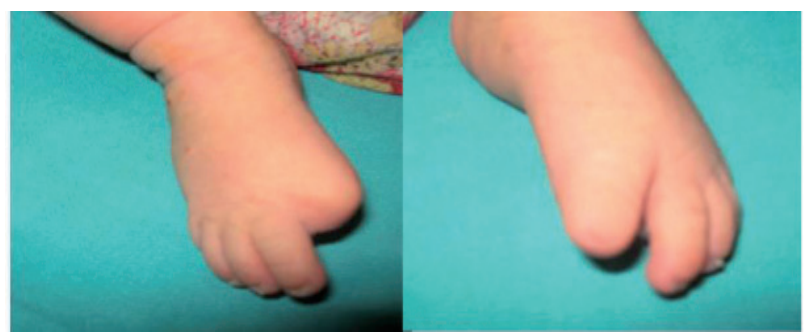

Figure 2. Both the great toes were amputated in utero. Entire right great toe was missing. The distal phalanx was missing in the left great toe.

In view of asymmetrical involvement, presence of amniotic bands, no other organ involvement and sporadic nature of occurrence, a diagnosis of amniotic band sequence was made. The constriction ring in the left middle finger was released and wound debridement carried out. The baby made an uneventful recovery and was discharged by 10 days of life and is on regular follow up. The parents have been advised regarding prosthesis and subsequent reconstructive surgery that the baby would need.

\section{DISCUSSION}

Amniotic Band Sequence is a group of congenital abnormalities caused by bands of amnion that attach to the fetus. ${ }^{2}$ Abnormalities result from attachment or constriction that lead to webbing of fingers and/or toes, amputation of limbs, severe defects of the head and face, spine, umbilical cord and/or body wall. In contrast, the term amniotic band syndrome encompasses many congenital anomalies viz. amniotic band disruption complex, amniochorionic meso-blastic fibrous strings, aberrant tissue bands, amniotic deformity, adhesions and mutilations (ADAM complex), amniotic adhesion malformation syndrome etc. ${ }^{1,6}$

Etiopathogenesis of $A B S$ remains in the realms of hypothesis. The "extrinsic model" proposed by Torpin and Faulkner in 1966 is now widely accepted. ${ }^{4}$ This theory explains the genesis of defects by rupture of the amnion in early pregnancy, with forming of amniotic bands and amniotic liquid loss, followed by extrusion of all or parts of the fetus into the chorionic cavity. Bands entrap parts of the growing fetus which become entangled and are subjected to compression with consequent compromise of fetal circulation and also its growth and development leading to disturbances of functions and anatomy. The alternative "intrinsic model" proposed by Streeter in 1930 suggests that the anomalies and the fibrous bands have a common origin, caused by a perturbation of developing germinal disc of the early embryo. ${ }^{5,6}$

Management includes early detection by serial ultrasound examination during pregnancy and intrauterine interventional procedures for maintaining distal vascularity. ${ }^{6}$ The indications for fetal surgery in the ABS may be either for a life-threatening condition viz. constriction of the umbilical cord, or more commonly, threatened limb amputation due to ABS when fetoscopic lysis of the amniotic band can be carried out. The earliest that amniotic bands have been detected was at 12 weeks gestation, by vaginal ultrasound. ${ }^{7}$ Bands may be difficult to detect by ultrasound, and are more often diagnosed by the effect they have on the fetal anatomy, as in the case of missing or misshapen limbs. Fetal MRI is able to visualize amniotic bands and their secondary manifestations and could be complementary to prenatal US when fetal surgery is contemplated. ${ }^{8}$ Plastic surgery may salvage the distal limb if deep grooves that encircle and limit vascular supply are treated in time. Once the distal part is gangrenous or amputated as in our baby, then reconstructive surgery and rehabilitation are to be carried out. Postnatal ultrasound of the brain and abdomen are important to detect structural defects due to vascular disruption. ${ }^{9}$ There may be hypoplasia of the lung due to oligohydramnios with respiratory insufficiency. If proper supportive care is rendered the outcome is usually good in most of the cases. Life expectancy is normal if visceral organs are spared. 
Congenital amputations should be distinguished from congenital hypoplasia and/or aplasia. The latter are due to transverse defects and hypoplasias are usually caused by monogenic mutations, are severely damaging to the fetus, with a high risk of recurrence (often $25 \%$ ).5,6 Risk of recurrence of amniotic band sequence is rare. An association with family history of Ehler Danlos and epidermolysis bullosa has been mentioned in literature. ${ }^{10}$ Differentiation is important in genetic counselling and evaluating the risk of recurrence.
Amniotic band sequence though a rather uncommon disorder can have serious and debilitating consequences on the neonate. Limb defects are the commonest. Our baby had a severe involvement of the extremities. A multidisciplinary approach involving early vascular decompression, reconstructive surgery, prosthesis and physiotherapy would help provide this hapless neonate with a functional rehabilitation.

\section{REFERENCES}

1. Foulkes GD, Reinker K. Congenital constriction and band syndrome: a seventy-year experience. J Pediatr Orthop. 1994; 14: 242-248.

2. Kalousek DK, Bamforth S. Amnion rupture sequence in previable fetuses. Am J Med Genet. 1988;1:63-73.

3. Robin NH, Franklin J, Prucka S, Ryan AB, Grant JH. Clefting, amniotic bands and polydactyly: A distinct phenotype that supports an intrinsic mechanism for amniotic band sequence. Am J Med Genet A. 2005;137 A(3):298-301.

4. Torpin R. Fetal Malformations Caused by Amnion Rupture During Gestation. Springfield, IL: Charles C Thomas; 1968.

5. Streeter GL. Focal deficiencies in fetal tissues and their relation to intrauterine amputations. Contrib Embryol Carnegie Inst. 1930;22:1-44.

6. Cignini P, Giorlandino C, Padula F, Dugo N, Cafà EV, Spata A. Epidemiology and risk factors of amniotic band syndrome, or ADAM sequence. J Prenat Med. 2012;6:59-63.

7. Allen LM, Silverman RK, Nosovitch JT, Lohnes TM, Williams KD. Constriction Rings and Congenital Amputations of the Fingers and Toes in a Mild Case of Amniotic Band Syndrome. J Diag Med Sonography. 2007; 23:280-285.

8. Neuman J, Calvo-Garcia MA, Kline-Fath BM, Bitters C, Merrow AC, Guimaraes CV, Lim FY. Prenatal imaging of amniotic band sequence: utility and role of fetal MRI as an adjunct to prenatal US. Pediatr Radiol. 2012;42:544-51.

9. Merrimen JL, McNeely PD, Bendor-Samuel RL, Schmidt $\mathrm{MH}$, Fraser RB. Congenital placental-cerebral adhesion: an unusual case of amniotic band sequence - Case report. J Neurosurg. 2006;104 (5 Suppl):352-5.

10. Abu Salah OT. Amniotic band sequence: A case report. RMJ 2011;36(2):159-162. 